Article

\title{
On the Most Extended Modal Operator of First Type over Interval-Valued Intuitionistic Fuzzy Sets
}

\author{
Krassimir Atanassov 1,2 (D) \\ 1 Department of Bioinformatics and Mathematical Modelling, Institute of Biophysics and Biomedical \\ Engineering, Bulgarian Academy of Sciences, 105 Acad. G. Bonchev Str., 1113 Sofia, Bulgaria; krat@bas.bg \\ 2 Intelligent Systems Laboratory, Prof. Asen Zlatarov University, 8010 Bourgas, Bulgaria
}

Received: 30 May 2018; Accepted: 4 July 2018; Published: 13 July 2018

\begin{abstract}
The definition of the most extended modal operator of first type over interval-valued intuitionistic fuzzy sets is given, and some of its basic properties are studied.
\end{abstract}

Keywords: interval-valued intuitionistic fuzzy set; intuitionistic fuzzy set; modal operator

\section{Introduction}

Intuitionistic fuzzy sets (IFSs; see [1-5]) were introduced in 1983 as an extension of the fuzzy sets defined by Lotfi Zadeh (4.2.1921-6.9.2017) in [6]. In recent years, the IFSs have also been extended: intuitionistic $L$-fuzzy sets [7], IFSs of second [8] and $n$th [9-12] types, temporal IFSs $[4,5,13]$, multidimensional IFSs $[5,14]$, and others. Interval-valued intuitionistic fuzzy sets (IVIFSs) are the most detailed described extension of IFSs. They appeared in 1988, when Georgi Gargov (7.4.1947-9.11.1996) and the author read Gorzalczany's paper [15] on the interval-valued fuzzy set (IVFS). The idea of IVIFS was announced in $[16,17]$ and extended in $[4,18]$, where the proof that IFSs and IVIFSs are equipollent generalizations of the notion of the fuzzy set is given.

Over IVIFS, many (more than the ones over IFSs) relations, operations, and operators are defined. Here, similar to the IFS case, the standard modal operators $\square$ and $\diamond$ have analogues, but their extensions-the intuitionistic fuzzy extended modal operators of the first type-already have two different forms. In the IFS case, there is an operator that includes as a partial case all other extended modal operators. In the present paper, we construct a similar operator for the case of IVIFSs and study its properties.

\section{Preliminaries}

Let us have a fixed universe $E$ and its subset $A$. The set

$$
A=\left\{\left\langle x, M_{A}(x), N_{A}(x)\right\rangle \mid x \in E\right\},
$$

where $M_{A}(x) \subset[0,1]$ and $N_{A}(x) \subset[0,1]$ are closed intervals and for all $x \in E$ :

$$
\sup M_{A}(x)+\sup N_{A}(x) \leq 1
$$

is called IVIFS, and functions $M_{A}: E \rightarrow \mathcal{P}([0,1])$ and $N_{A}: E \rightarrow \mathcal{P}([0,1])$ represent the set of degrees of membership (validity, etc.) and the set of degrees of non-membership (non-validity, etc.) of element $x \in E$ to a fixed set $A \subseteq E$, where $\mathcal{P}(Z)=\{Y \mid Y \subseteq Z\}$ for an arbitrary set $Z$.

Obviously, both intervals have the representation:

$$
M_{A}(x)=\left[\inf M_{A}(x), \sup M_{A}(x)\right],
$$




$$
N_{A}(x)=\left[\inf N_{A}(x), \sup N_{A}(x)\right]
$$

Therefore, when

$$
\inf M_{A}(x)=\sup M_{A}(x)=\mu_{A}(x) \text { and } \inf N_{A}(x)=\sup N_{A}(x)=v_{A}(x),
$$

the IVIFS $A$ is transformed to an IFS.

We must mention that in $[19,20]$ the second geometrical interpretation of the IFSs is given (see Figure 1).

IVIFSs have geometrical interpretations similar to, but more complex than, those of the IFSs. For example, the analogue of the geometrical interpretation from Figure 1 is shown in Figure 2.

Obviously, each IVFS $A$ can be represented by an IVIFS as

$$
\begin{gathered}
A=\left\{\left\langle x, M_{A}(x), N_{A}(x)\right\rangle \mid x \in E\right\} \\
=\left\{\left\langle x, M_{A}(x),\left[1-\sup M_{A}(x), 1-\inf M_{A}(x)\right]\right\rangle \mid x \in E\right\} .
\end{gathered}
$$

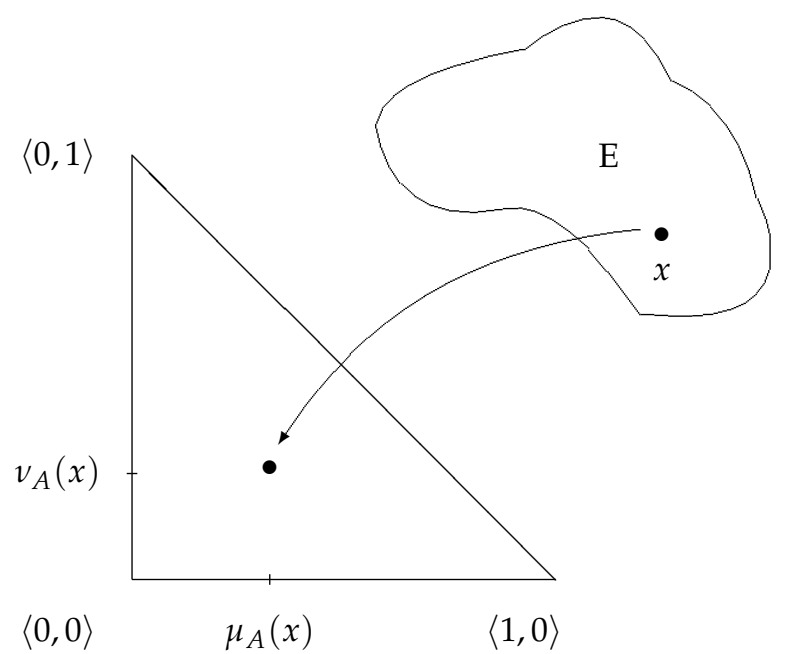

Figure 1. The second geometrical interpretation of an intuitionistic fuzzy set (IFS).

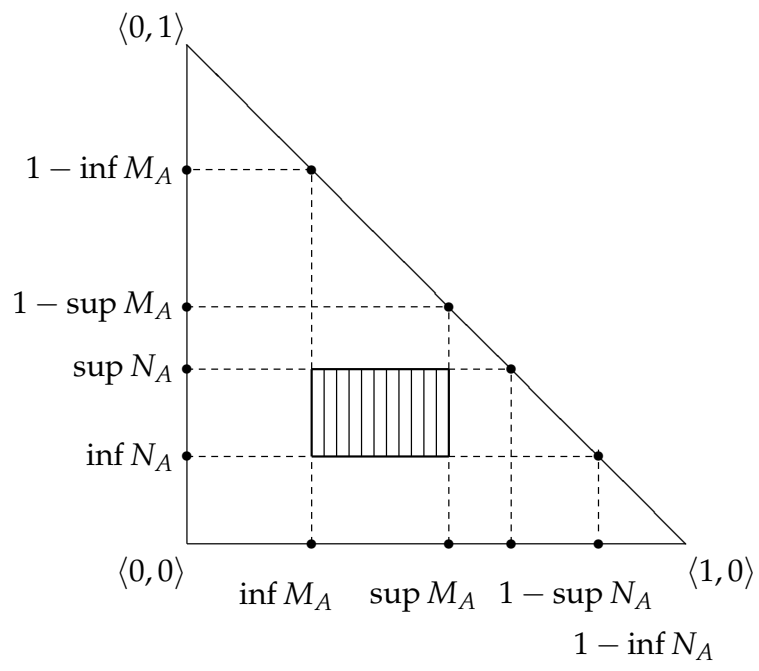

Figure 2. The second geometrical interpretation of an interval-valued intuitionistic fuzzy set (IVIFS). 
The geometrical interpretation of the IVFS $A$ is shown in Figure 3. It has the form of a section lying on the triangle's hypotenuse.

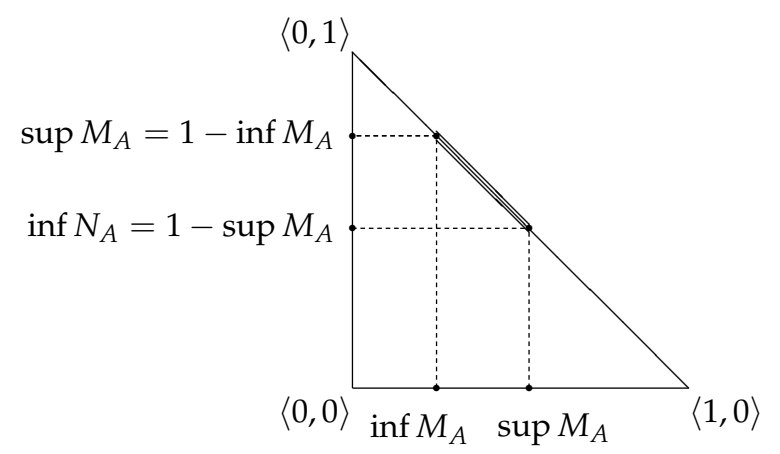

Figure 3. The second geometrical interpretation of an IVFS.

Modal-type operators are defined similarly to those defined for IFSs, but here they have two forms: shorter and longer. The shorter form is:

$$
\begin{aligned}
\square A= & \left\{\left\langle x, M_{A}(x),\left[\inf N_{A}(x), 1-\sup M_{A}(x)\right]\right\rangle \mid x \in E\right\}, \\
\nabla A= & \left\{\left\langle x,\left[\inf M_{A}(x), 1-\sup N_{A}(x)\right], N_{A}(x)\right\rangle \mid x \in E\right\}, \\
D_{\alpha}(A)= & \left\{\left\langlex,\left[\inf M_{A}(x), \sup M_{A}(x)+\alpha\left(1-\sup M_{A}(x)-\sup N_{A}(x)\right)\right],\right.\right. \\
& {\left.\left[\inf N_{A}(x), \sup N_{A}(x)+(1-\alpha)\left(1-\sup M_{A}(x)-\sup N_{A}(x)\right)\right]\right\rangle } \\
& \mid x \in E\}, \\
F_{\alpha, \beta}(A)= & \left\{\left\langlex,\left[\inf M_{A}(x), \sup M_{A}(x)+\alpha\left(1-\sup M_{A}(x)-\sup N_{A}(x)\right)\right],\right.\right. \\
& {\left.\left[\inf N_{A}(x), \sup N_{A}(x)+\beta\left(1-\sup M_{A}(x)-\sup N_{A}(x)\right)\right]\right\rangle } \\
& \mid x \in E\}, \text { for } \alpha+\beta \leq 1, \\
G_{\alpha, \beta}(A)= & \left\{\left\langle x,\left[\alpha \inf M_{A}(x), \alpha \sup M_{A}(x)\right],\left[\beta \inf N_{A}(x), \beta \sup N_{A}(x)\right]\right\rangle\right. \\
& \mid x \in E\}, \\
H_{\alpha, \beta}(A)= & \left\{\left\langlex,\left[\alpha \inf M(x), \alpha \sup M_{A}(x)\right],\left[\inf N_{A}(x), \sup N_{A}(x)\right.\right.\right. \\
& \left.\left.\left.+\beta\left(1-\sup M_{A}(x)-\sup N_{A}(x)\right)\right]\right\rangle \mid x \in E\right\}, \\
H_{\alpha, \beta}^{*}(A)= & \left\{\left\langlex,\left[\alpha \inf M_{A}(x), \alpha \sup M_{A}(x)\right],\left[\inf N_{A}(x), \sup N_{A}(x)\right.\right.\right. \\
& \left.\left.\left.+\beta\left(1-\alpha \sup M_{A}(x)-\sup N_{A}(x)\right)\right]\right\rangle \mid x \in E\right\}, \\
J_{\alpha, \beta}(A)= & \left\{\left\langlex,\left[\inf M_{A}(x), \sup M_{A}(x)+\alpha\left(1-\sup M_{A}(x)\right.\right.\right.\right. \\
& \left.\left.\left.\left.-\sup N_{A}(x)\right)\right],\left[\beta \inf N_{A}(x), \beta \sup N_{A}(x)\right]\right\rangle \mid x \in E\right\}, \\
J_{\alpha, \beta}^{*}(A)= & \left\{\left\langlex,\left[\inf M_{A}(x), \sup M_{A}(x)+\alpha\left(1-\sup M_{A}(x)\right.\right.\right.\right. \\
& \left.\left.\left.\left.-\beta \sup N_{A}(x)\right)\right],\left[\beta \inf N_{A}(x), \beta \cdot \sup N_{A}(x)\right]\right\rangle \mid x \in E\right\},
\end{aligned}
$$

where $\alpha, \beta \in[0,1]$.

Obviously, as in the case of IFSs, the operator $D_{\alpha}$ is an extension of the intuitionistic fuzzy forms of (standard) modal logic operators $\square$ and $\diamond$, and it is a partial case of $F_{\alpha, \beta}$. 
The longer form of these operators (operators $\square, \diamond$, and $D$ do not have two forms—only the one above) is (see [4]):

$$
\begin{aligned}
& \bar{F}_{\left(\begin{array}{ll}
\alpha & \gamma \\
\beta & \delta
\end{array}\right)}(A)=\left\{\left\langlex,\left[\inf M_{A}(x)+\alpha\left(1-\sup M_{A}(x)-\sup N_{A}(x)\right),\right.\right.\right. \\
& \left.\sup M_{A}(x)+\beta\left(1-\sup M_{A}(x)-\sup N_{A}(x)\right)\right], \\
& {\left[\inf N_{A}(x)+\gamma\left(1-\sup M_{A}(x)-\sup N_{A}(x)\right)\right. \text {, }} \\
& \left.\left.\left.\sup N_{A}(x)+\delta\left(1-\sup M_{A}(x)-\sup N_{A}(x)\right)\right]\right\rangle \mid x \in E\right\} \\
& \text { where } \beta+\delta \leq 1 \text {, } \\
& \bar{G}_{\left(\begin{array}{ll}
\alpha & \gamma \\
\beta & \delta
\end{array}\right)}(A)=\left\{\left\langlex,\left[\alpha \inf M_{A}(x), \beta \sup M_{A}(x)\right],\right.\right. \\
& \left.\left.\left[\gamma \inf N_{A}(x), \delta \sup N_{A}(x)\right]\right\rangle \mid x \in E\right\}, \\
& \bar{H}_{\left(\begin{array}{ll}
\alpha & \gamma \\
\beta & \delta
\end{array}\right)}(A)=\left\{\left\langlex,\left[\alpha \inf M_{A}(x), \beta \sup M_{A}(x)\right]\right.\right. \text {, } \\
& {\left[\inf N_{A}(x)+\gamma\left(1-\sup M_{A}(x)-\sup N_{A}(x)\right),\right.} \\
& \left.\left.\left.\sup N_{A}(x)+\delta\left(1-\sup M_{A}(x)-\sup N_{A}(x)\right)\right]\right\rangle \mid x \in E\right\}, \\
& \bar{H}_{\left(\begin{array}{cc}
\alpha & \gamma \\
\beta & \delta
\end{array}\right)}^{*}(A)=\left\{\left\langlex,\left[\alpha \inf M_{A}(x), \beta \sup M_{A}(x)\right]\right.\right. \text {, } \\
& {\left[\inf N_{A}(x)+\gamma\left(1-\beta \sup M_{A}(x)-\sup N_{A}(x)\right),\right.} \\
& \left.\left.\left.\sup N_{A}(x)+\delta\left(1-\beta \sup M_{A}(x)-\sup N_{A}(x)\right)\right]\right\rangle \mid x \in E\right\}, \\
& \bar{J}_{\left(\begin{array}{ll}
\alpha & \gamma \\
\beta & \delta
\end{array}\right)}=\left\{\left\langlex,\left[\inf M_{A}(x)+\alpha\left(1-\sup M_{A}(x)-\sup N_{A}(x)\right)\right. \text {, }\right.\right. \\
& \left.\sup M_{A}(x)+\beta\left(1-\sup M_{A}(x)-\sup N_{A}(x)\right)\right], \\
& \left.\left.\left[\gamma \inf N_{A}(x), \delta \sup N_{A}(x)\right]\right\rangle \mid x \in E\right\}, \\
& \bar{J}_{\left(\begin{array}{ll}
\alpha & \gamma \\
\beta & \delta
\end{array}\right)}(A)=\left\{\left\langlex,\left[\inf M_{A}(x)+\alpha\left(1-\delta \sup M_{A}(x)-\sup N_{A}(x)\right),\right.\right.\right. \\
& \left.\sup M_{A}(x)+\beta\left(1-\sup M_{A}(x)-\delta \sup N_{A}(x)\right)\right], \\
& \left.\left.\left[\gamma \cdot \inf N_{A}(x), \delta \cdot \sup N_{A}(x)\right]\right\rangle \mid x \in E\right\},
\end{aligned}
$$

where $\alpha, \beta, \gamma, \delta \in[0,1]$ such that $\alpha \leq \beta$ and $\gamma \leq \delta$.

Figure 4 shows to which region of the triangle the element $x \in E$ (represented by the small rectangular region in the triangle) will be transformed by the operators $F, G, \ldots$, irrespective of whether they have two or four indices.

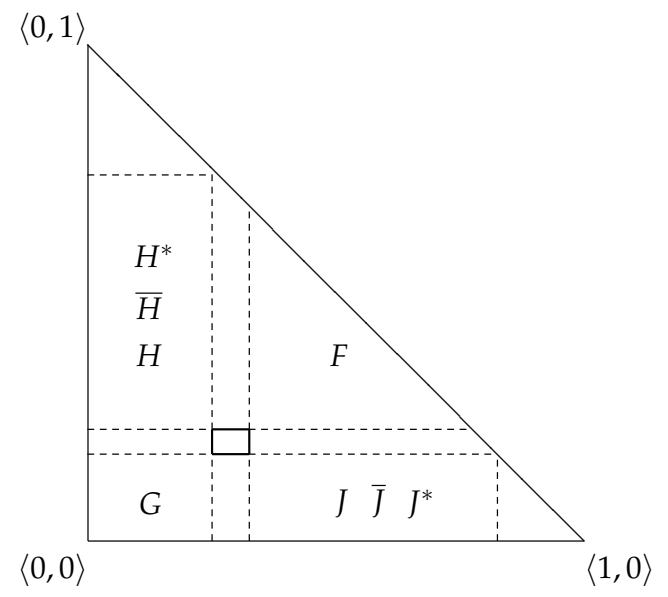

Figure 4. Region of transformation by the application of the operators. 


\section{Operator $X$}

Now, we introduce the new operator

$$
\begin{aligned}
& X\left(\begin{array}{llllll}
a_{1} & b_{1} & c_{1} & d_{1} & e_{1} & f_{1} \\
a_{2} & b_{2} & c_{2} & d_{2} & e_{2} & f_{2}
\end{array}\right)(A) \\
& =\left\{\left\langlex,\left[a_{1} \inf M_{A}(x)+b_{1}\left(1-\inf M_{A}(x)-c_{1} \inf N_{A}(x)\right),\right.\right.\right. \\
& \left.a_{2} \sup M_{A}(x)+b_{2}\left(1-\sup M_{A}(x)-c_{2} \sup N_{A}(x)\right)\right] \text {, } \\
& {\left[d_{1} \inf N_{A}(x)+e_{1}\left(1-f_{1} \inf M_{A}(x)-\inf N_{A}(x)\right),\right.} \\
& \left.\left.\left.d_{2} \sup N_{A}(x)+e_{2}\left(1-f_{2} \sup M_{A}(x)-\sup N_{A}(x)\right)\right]\right\rangle \mid x \in E\right\},
\end{aligned}
$$

where $a_{1}, b_{1}, c_{1}, d_{1}, e_{1}, f_{1}, a_{2}, b_{2}, c_{2}, d_{2}, e_{2}, f_{2} \in[0,1]$, the following three conditions are valid for $i=1,2$ :

$$
\begin{gathered}
a_{i}+e_{i}-e_{i} f_{i} \leq 1, \\
b_{i}+d_{i}-b_{i} c_{i} \leq 1, \\
b_{i}+e_{i} \leq 1,
\end{gathered}
$$

and

$$
a_{1} \leq a_{2}, b_{1} \leq b_{2}, c_{1} \leq c_{2}, d_{1} \leq d_{2}, e_{1} \leq e_{2}, f_{1} \leq f_{2}
$$

Theorem 1. For every IVIFS $A$ and for every $a_{1}, b_{1}, c_{1}, d_{1}, e_{1}, f_{1}, a_{2}, b_{2}, c_{2}, d_{2}, e_{2}, f_{2} \in[0,1]$ that satisfy (2)-(5), $X\left(\begin{array}{llllll}a_{1} & b_{1} & c_{1} & d_{1} & e_{1} & f_{1} \\ a_{2} & b_{2} & c_{2} & d_{2} & e_{2} & f_{2}\end{array}\right)(A)$ is an IVIFS.

Proof. Let $a_{1}, b_{1}, c_{1}, d_{1}, e_{1}, f_{1}, a_{2}, b_{2}, c_{2}, d_{2}, e_{2}, f_{2} \in[0,1]$ satisfy (2)-(5) and let $A$ be a fixed IVIFS. Then, from (5) it follows that

$$
\begin{aligned}
& a_{1} \inf M_{A}(x)+b_{1}\left(1-\inf M_{A}(x)-c_{1} \inf N_{A}(x)\right) \\
\leq & a_{2} \sup M_{A}(x)+b_{2}\left(1-\sup M_{A}(x)-c_{2} \sup N_{A}(x)\right)
\end{aligned}
$$

and

$$
\begin{aligned}
& d_{1} \inf N_{A}(x)+e_{1}\left(1-f_{1} \inf M_{A}(x)-\inf N_{A}(x)\right) \\
\leq & d_{2} \sup N_{A}(x)+e_{2}\left(1-f_{2} \sup M_{A}(x)-\sup N_{A}(x)\right) .
\end{aligned}
$$

Now, from (5) it is clear that it will be enough to check that

$$
\begin{gathered}
X=a_{2} \sup M_{A}(x)+b_{2}\left(1-\sup M_{A}(x)-c_{2} \sup N_{A}(x)\right) \\
+d_{2} \sup N_{A}(x)+e_{2}\left(1-f_{2} \sup M_{A}(x)-\sup N_{A}(x)\right) \\
=\left(a_{2}-b_{2}-e_{2} f_{2}\right) \sup M_{A}(x)+\left(d_{2}-e_{2}-b_{2} c_{2}\right) \sup N_{A}(x)+b_{2}+e_{2} \leq 1 .
\end{gathered}
$$

In fact, from (2),

$$
a_{2}-b_{2}-e_{2} f_{2} \leq 1-b_{2}-e_{2}
$$

and from (3):

$$
d_{2}-e_{2}-b_{2} c_{2} \leq 1-b_{2}-e_{2}
$$

Then, from (1),

$$
X \leq\left(1-b_{2}-e_{2}\right)\left(\sup M_{A}(x)+\sup N_{A}(x)\right)+b_{2}+e_{2}
$$




$$
\leq 1-b_{2}-e_{2}+b_{2}+e_{2}=1 \text {. }
$$

Finally, when $\sup M_{A}(x)=\inf N_{A}(x)=0$ and from (4),

$$
X=b_{2}(1-0-0)+e_{2}(1-0-0)=b_{2}+e_{2} \leq 1 .
$$

Therefore, the definition of the IVIFS is correct.

All of the operators described above can be represented by the operator $X\left(\begin{array}{llllll}a_{1} & b_{1} & c_{1} & d_{1} & e_{1} & f_{1} \\ a_{2} & b_{2} & c_{2} & d_{2} & e_{2} & f_{2}\end{array}\right)$ at suitably chosen values of its parameters. These representations are the following:

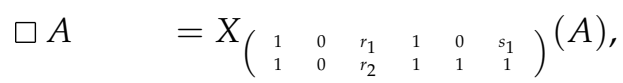

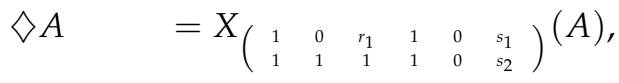

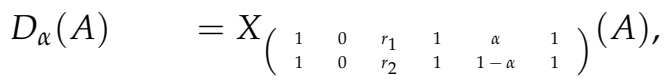

$$
\begin{aligned}
& F_{\alpha, \beta}(A)=X\left(\begin{array}{cccccc}
1 & 0 & r_{1} & 1 & 0 & s_{1} \\
1 & \alpha & 1 & 1 & \beta & 1
\end{array}\right)(A) \text {, } \\
& G_{\alpha, \beta}(A)=X\left(\begin{array}{llllll}
\alpha & 0 & r_{1} & \beta & 0 & s_{1} \\
\alpha & 0 & r_{2} & \beta & 0 & s_{2}
\end{array}\right)(A), \\
& H_{\alpha, \beta}(A)=X\left(\begin{array}{llllll}
\alpha & 0 & r_{1} & 1 & 0 & s_{1} \\
\alpha & 0 & r_{2} & 1 & \beta & 1
\end{array}\right)(A), \\
& H_{\alpha, \beta}^{*}(A)=X\left(\begin{array}{cccccc}
\alpha & 0 & r_{1} & \alpha & 0 & s_{1} \\
1 & 0 & r_{2} & 1 & \beta & \alpha
\end{array}\right)(A), \\
& J_{\alpha, \beta}(A) \quad=X\left(\begin{array}{cccccc}
1 & 0 & r_{1} & \beta & 0 & s_{1} \\
1 & \alpha & 1 & \beta & 0 & s_{2}
\end{array}\right)(A), \\
& J_{\alpha, \beta}^{*}(A)=X\left(\begin{array}{cccccc}
1 & 0 & r_{1} & \beta & 0 & s_{1} \\
1 & \alpha & \beta & \beta & 0 & s_{2}
\end{array}\right)(A), \\
& \bar{F}\left(\begin{array}{ll}
\alpha & \gamma \\
\beta & \delta
\end{array}\right)(A)=X\left(\begin{array}{cccccc}
1 & \alpha & 1 & 1 & \gamma & 1 \\
1 & \beta & 1 & 1 & \delta & s_{1}
\end{array}\right)(A),
\end{aligned}
$$

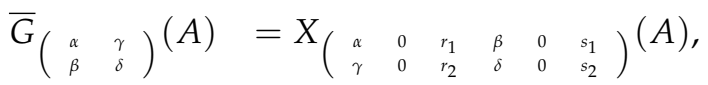

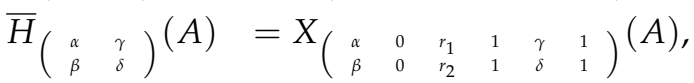

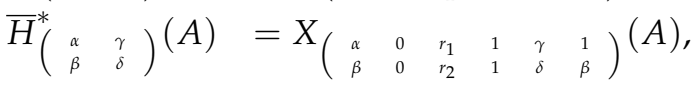

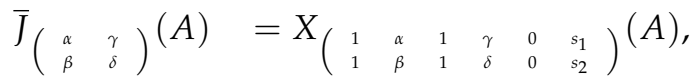

$$
\begin{aligned}
& \bar{J}_{\left(\begin{array}{ll}
\alpha \\
\beta & \gamma \\
\beta & \delta
\end{array}\right)}(A)=X\left(\begin{array}{llllll}
1 & \alpha & \delta & \gamma & 0 & s_{1} \\
1 & \beta & \delta & \delta & 0 & s_{2}
\end{array}\right)(A),
\end{aligned}
$$

where $r_{1}, r_{2}, s_{1}, s_{2}$ are arbitrary real numbers in the interval $[0,1]$.

Three of the operations, defined over two IVIFSs $A$ and $B$, are the following:

$$
\begin{aligned}
\neg A= & \left\{\left\langle x, N_{A}(x), M_{A}(x)\right\rangle \mid x \in E\right\}, \\
A \cap B= & \left\{\left\langlex,\left[\min \left(\inf M_{A}(x), \inf M_{B}(x)\right), \min \left(\sup M_{A}(x), \sup M_{B}(x)\right)\right],\right.\right. \\
& {\left.\left.\left[\max \left(\inf N_{A}(x), \inf N_{B}(x)\right), \max \left(\sup N_{A}(x), \sup N_{B}(x)\right)\right]\right\rangle \mid x \in E\right\}, } \\
A \cup B= & \left\{\left\langlex,\left[\max \left(\inf M_{A}(x), \inf M_{B}(x)\right), \max \left(\sup M_{A}(x), \sup M_{B}(x)\right)\right],\right.\right. \\
& {\left.\left.\left[\min \left(\inf N_{A}(x), \inf N_{B}(x)\right), \min \left(\sup N_{A}(x), \sup N_{B}(x)\right)\right]\right\rangle \mid x \in E\right\} . }
\end{aligned}
$$


For any two IVIFSs $A$ and $B$, the following relations hold:

$$
\begin{aligned}
A \subset B \quad \text { iff } \quad \forall x \in E, \inf M_{A}(x) \leq \inf M_{B}(x), \inf N_{A}(x) \geq \inf N_{B}(x), \\
\left.\quad \sup M_{A}(x) \leq \sup M_{B}(x) \text { and } \sup N_{A}(x) \geq \sup N_{B}(x)\right), \\
A \supset B \text { iff } \quad B \subset A, \\
A=B \quad \text { iff } \quad A \subset B \text { and } B \subset A .
\end{aligned}
$$

Theorem 2. For every two IVIFSs $A$ and $B$ and for every $a_{1}, b_{1}, c_{1}, d_{1}, e_{1}, f_{1}, a_{2}, b_{2}, c_{2}, d_{2}, e_{2}, f_{2} \in[0,1]$ that satisfy (2)-(5),

$$
\begin{aligned}
& \text { (a) } \neg X\left(\begin{array}{llllll}
a_{1} & b_{1} & c_{1} & d_{1} & e_{1} & f_{1} \\
a_{2} & b_{2} & c_{2} & d_{2} & e_{2} & f_{2}
\end{array}\right)(\neg A)=X_{d, e, f, a, b, c}(A) \text {, } \\
& \text { (b) } X\left(\begin{array}{llllll}
a_{1} & b_{1} & c_{1} & d_{1} & e_{1} & f_{1} \\
a_{2} & b_{2} & c_{2} & d_{2} & e_{2} & f_{2}
\end{array}\right)(A \cap B)
\end{aligned}
$$

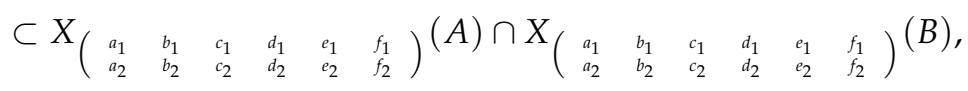

$$
\begin{aligned}
& \text { (c) } X\left(\begin{array}{llllll}
a_{1} & b_{1} & c_{1} & d_{1} & e_{1} & f_{1} \\
a_{2} & b_{2} & c_{2} & d_{2} & e_{2} & f_{2}
\end{array}\right)(A \cup B) \\
& \supset X\left(\begin{array}{llllll}
a_{1} & b_{1} & c_{1} & d_{1} & e_{1} & f_{1} \\
a_{2} & b_{2} & c_{2} & d_{2} & e_{2} & f_{2}
\end{array}\right)(A) \cup X\left(\begin{array}{llllll}
a_{1} & b_{1} & c_{1} & d_{1} & e_{1} & f_{1} \\
a_{2} & b_{2} & c_{2} & d_{2} & e_{2} & f_{2}
\end{array}\right)(B) .
\end{aligned}
$$

Proof. (c) Let $a_{1}, b_{1}, c_{1}, d_{1}, e_{1}, f_{1}, a_{2}, b_{2}, c_{2}, d_{2}, e_{2}, f_{2} \in[0,1]$ satisfy (2)-(5), and let $A$ and $B$ be fixed IVIFSs. First, we obtain:

$$
\begin{aligned}
& Y=X\left(\begin{array}{llllll}
a_{1} & b_{1} & c_{1} & d_{1} & e_{1} & f_{1} \\
a_{2} & b_{2} & c_{2} & d_{2} & e_{2} & f_{2}
\end{array}\right)(A \cup B) \\
& =X\left(\begin{array}{llllll}
a_{1} & b_{1} & c_{1} & d_{1} & e_{1} & f_{1} \\
a_{2} & b_{2} & c_{2} & d_{2} & e_{2} & f_{2}
\end{array}\right)\left(\left\{\left\langlex,\left[\max \left(\inf M_{A}(x), \inf M_{B}(x)\right),\right.\right.\right.\right. \\
& \left.\max \left(\sup M_{A}(x), \sup M_{B}(x)\right)\right] \\
& \left.\left.\left.\left[\min \left(\inf N_{A}(x), \inf N_{B}(x)\right), \min \left(\sup N_{A}(x), \sup N_{B}(x)\right)\right]\right\rangle \mid x \in E\right\}\right) \\
& =\left\{\left\langlex,\left[a_{1} \max \left(\inf M_{A}(x), \inf M_{B}(x)\right)+b_{1}\left(1-\max \left(\inf M_{A}(x), \inf M_{B}(x)\right)\right.\right.\right.\right. \\
& \left.-c_{1} \min \left(\inf N_{A}(x), \inf N_{B}(x)\right)\right), a_{2} \max \left(\sup M_{A}(x) \sup M_{B}(x)\right) \\
& \left.+b_{2}\left(1-\max \left(\sup M_{A}(x) \sup M_{B}(x)\right)-c_{2} \min \left(\sup N_{A}(x), \sup N_{B}(x)\right)\right)\right], \\
& {\left[d_{1} \min \left(\inf N_{A}(x), \inf N_{B}(x)\right)+e_{1}\left(1-f_{1} \max \left(\inf M_{A}(x), \inf M_{B}(x)\right)\right.\right.} \\
& \left.-\min \left(\inf N_{A}(x), \inf N_{B}(x)\right)\right), d_{2} \min \left(\sup N_{A}(x), \sup N_{B}(x)\right) \\
& \left.\left.\left.+e_{2}\left(1-f_{2} \max \left(\sup M_{A}(x) \sup M_{B}(x)\right)-\min \left(\sup N_{A}(x), \sup N_{B}(x)\right)\right)\right]\right\rangle \mid x \in E\right\} \text {. }
\end{aligned}
$$

Second, we calculate:

$$
\begin{aligned}
& Z=X\left(\begin{array}{llllll}
a_{1} & b_{1} & c_{1} & d_{1} & e_{1} & f_{1} \\
a_{2} & b_{2} & c_{2} & d_{2} & e_{2} & f_{2}
\end{array}\right)(A) \cup X\left(\begin{array}{llllll}
a_{1} & b_{1} & c_{1} & d_{1} & e_{1} & f_{1} \\
a_{2} & b_{2} & c_{2} & d_{2} & e_{2} & f_{2}
\end{array}\right)(B) \\
& =\left\{\left\langlex,\left[a_{1} \inf M_{A}(x)+b_{1}\left(1-\inf M_{A}(x)-c_{1} \inf N_{A}(x)\right),\right.\right.\right. \\
& \left.a_{2} \sup M_{A}(x)+b_{2}\left(1-\sup M_{A}(x)-c_{2} \sup N_{A}(x)\right)\right] \text {, } \\
& {\left[d_{1} \inf N_{A}(x)+e_{1}\left(1-f_{1} \inf M_{A}(x)-\inf N_{A}(x)\right),\right.} \\
& \left.\left.\left.d_{2} \sup N_{A}(x)+e_{2}\left(1-f_{2} \sup M_{A}(x)-\sup N_{A}(x)\right)\right]\right\rangle \mid x \in E\right\}
\end{aligned}
$$




$$
\begin{gathered}
\cup\left\{\left\langlex,\left[a_{1} \inf M_{B}(x)+b_{1}\left(1-\inf M_{B}(x)-c_{1} \inf N_{B}(x)\right),\right.\right.\right. \\
\left.a_{2} \sup M_{B}(x)+b_{2}\left(1-\sup M_{B}(x)-c_{2} \sup N_{B}(x)\right)\right], \\
{\left[d_{1} \inf N_{B}(x)+e_{1}\left(1-f_{1} \inf M_{B}(x)-\inf N_{B}(x)\right),\right.} \\
\left.\left.\left.d_{2} \sup N_{B}(x)+e_{2}\left(1-f_{2} \sup M_{B}(x)-\sup N_{B}(x)\right)\right]\right\rangle \mid x \in E\right\} \\
=\left\{\left\langlex,\left[\operatorname { m a x } \left(a_{1} \inf M_{A}(x)+b_{1}\left(1-\inf M_{A}(x)-c_{1} \inf N_{A}(x)\right),\right.\right.\right.\right. \\
\left.a_{1} \inf M_{B}(x)+b_{1}\left(1-\inf M_{B}(x)-c_{1} \inf N_{B}(x)\right)\right), \\
\max \left(a_{2} \sup M_{A}(x)+b_{2}\left(1-\sup M_{A}(x)-c_{2} \sup N_{A}(x)\right),\right. \\
\left.\left.a_{2} \sup M_{B}(x)+b_{2}\left(1-\sup M_{B}(x)-c_{2} \sup N_{B}(x)\right)\right)\right], \\
{\left[\operatorname { m i n } \left(d_{1} \inf N_{A}(x)+e_{1}\left(1-f_{1} \inf M_{A}(x)-\inf N_{A}(x)\right),\right.\right.} \\
\left.d_{1} \inf N_{B}(x)+e_{1}\left(1-f_{1} \inf M_{B}(x)-\inf N_{B}(x)\right)\right), \\
\min \left(d_{2} \sup N_{A}(x)+e_{2}\left(1-f_{2} \sup M_{A}(x)-\sup N_{A}(x)\right),\right. \\
\left.\left.\left.\left.d_{2} \sup N_{B}(x)+e_{2}\left(1-f_{2} \sup M_{B}(x)-\sup N_{B}(x)\right)\right)\right]\right\rangle \mid x \in E\right\} .
\end{gathered}
$$

Let

$$
\begin{gathered}
P=a_{1} \max \left(\inf M_{A}(x), \inf M_{B}(x)\right)+b_{1}\left(1-\max \left(\inf M_{A}(x), \inf M_{B}(x)\right)\right. \\
\left.-c_{1} \min \left(\inf N_{A}(x), \inf N_{B}(x)\right)\right)-\max \left(a_{1} \inf M_{A}(x)+b_{1}\left(1-\inf M_{A}(x)-c_{1} \inf N_{A}(x)\right),\right. \\
\left.a_{1} \inf M_{B}(x)+b_{1}\left(1-\inf M_{B}(x)-c_{1} \inf N_{B}(x)\right)\right) \\
=a_{1} \max \left(\inf M_{A}(x), \inf M_{B}(x)\right)+b_{1}-b_{1} \max \left(\inf M_{A}(x), \inf M_{B}(x)\right) \\
\left.-b_{1} c_{1} \min \left(\inf N_{A}(x), \inf N_{B}(x)\right)\right)-\max \left(\left(a_{1}-b_{1}\right) \inf M_{A}(x)+b_{1}-b_{1} c_{1} \inf N_{A}(x),\right. \\
\left.\left(a_{1}-b_{1}\right) \inf M_{B}(x)+b_{1}-b_{1} c_{1} \inf N_{B}(x)\right) \\
=a_{1} \max \left(\inf M_{A}(x), \inf M_{B}(x)\right)-b_{1} \max \left(\inf M_{A}(x), \inf M_{B}(x)\right) \\
-b_{1} c_{1} \min \left(\inf N_{A}(x), \inf N_{B}(x)\right)-\max \left(\left(a_{1}-b_{1}\right) \inf M_{A}(x)-b_{1} c_{1} \inf N_{A}(x),\right. \\
\left.\left(a_{1}-b_{1}\right) \inf M_{B}(x)-b_{1} c_{1} \inf N_{B}(x)\right) .
\end{gathered}
$$

Let $\inf M_{A}(x) \geq \inf M_{B}(x)$. Then

$$
\begin{gathered}
P=\left(a_{1}-b_{1}\right) \inf M_{A}(x)-b_{1} c_{1} \min \left(\inf N_{A}(x), \inf N_{B}(x)\right)-\max \left(\left(a_{1}-b_{1}\right) \inf M_{A}(x)\right. \\
\left.-b_{1} c_{1} \inf N_{A}(x),\left(a_{1}-b_{1}\right) \inf M_{B}(x)-b_{1} c_{1} \inf N_{B}(x)\right) .
\end{gathered}
$$

Let $\left(a_{1}-b_{1}\right) \inf M_{A}(x)-b_{1} c_{1} \inf N_{A}(x) \geq\left(a_{1}-b_{1}\right) \inf M_{B}(x)-b_{1} c_{1} \inf N_{B}(x)$. Then

$$
\begin{gathered}
P=\left(a_{1}-b_{1}\right) \inf M_{A}(x)-b_{1} c_{1} \min \left(\inf N_{A}(x), \inf N_{B}(x)\right)-\left(a_{1}-b_{1}\right) \inf M_{A}(x) \\
+b_{1} c_{1} \inf N_{A}(x) \\
=b_{1} c_{1} \inf N_{A}(x)-b_{1} c_{1} \min \left(\inf N_{A}(x), \inf N_{B}(x)\right) \geq 0 .
\end{gathered}
$$

If $\left(a_{1}-b_{1}\right) \inf M_{A}(x)-b_{1} c_{1} \inf N_{A}(x)<\left(a_{1}-b_{1}\right) \inf M_{B}(x)-b_{1} c_{1} \inf N_{B}(x)$. Then

$$
P=\left(a_{1}-b_{1}\right) \inf M_{A}(x)-b_{1} c_{1} \min \left(\inf N_{A}(x), \inf N_{B}(x)\right)-\left(a_{1}-b_{1}\right) \inf M_{B}(x)
$$




$$
\begin{gathered}
\left.+b_{1} c_{1} \inf N_{B}(x)\right) . \\
=b_{1} c_{1} \inf N_{B}(x)-b_{1} c_{1} \min \left(\inf N_{A}(x), \inf N_{B}(x)\right) \geq 0 .
\end{gathered}
$$

Therefore, the $\inf M_{A}$-component of IVIFS $Y$ is higher than or equal to the $\inf M_{A}$-component of IVIFS Z. In the same manner, it can be checked that the same inequality is valid for the sup $M_{A}$-components of these IVIFSs. On the other hand, we can check that that the $\inf N_{A^{-}}$ and $\sup N_{A}$-components of IVIFS $Y$ are, respectively, lower than or equal to the $\inf N_{A}$ and sup $N_{A}$-components of IVIFS $Z$. Therefore, the inequality (c) is valid.

\section{Conclusions}

In the near future, the author plans to study some other properties of the new operator

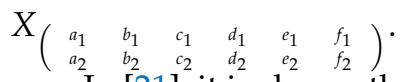

In [21], it is shown that the IFSs are a suitable tool for the evaluation of data mining processes and objects. In the near future, we plan to discuss the possibilities of using IVIFSs as a similar tool.

Funding: This research was funded by the Bulgarian National Science Fund under Grant Ref. No. DN-02-10/2016.

Conflicts of Interest: The author declares no conflict of interest.

\section{References}

1. Atanassov, K. Intuitionistic Fuzzy Sets. 1983, VII ITKR Session. Deposed in Centr. Sci.-Techn. Library of the Bulg. Acad. of Sci., 1697/84. Available online: http://www.biomed.bas.bg/bioautomation/2016/vol_20.s1/ files/20.s1_03.pdf (accessed on 13 July 2018)

2. Atanassov, K. Intuitionistic fuzzy sets. Int. J. Bioautom. 2016, 20, S1-S6.

3. Atanassov, K. Intuitionistic fuzzy sets. Fuzzy Sets Syst. 1986, 20, 87-96. [CrossRef]

4. Atanassov, K. Intuitionistic Fuzzy Sets; Springer: Heidelberg, Germany, 1999.

5. Atanassov, K. On Intuitionistic Fuzzy Sets Theory; Springer: Berlin, Germany, 2012.

6. Zadeh, L. Fuzzy sets. Inf. Control 1965, 8, 338-353. [CrossRef]

7. Atanassov, K.; Stoeva, S. Intuitionistic L-fuzzy sets. In Cybernetics and Systems Research 2; Trappl, R., Ed.; Elsevier Sci. Publ.: Amsterdam, The Nertherlands, 1984; pp. 539-540.

8. Atanassov, K. A second type of intuitionistic fuzzy sets. BUSEFAL 1993, 56, 66-70.

9. Atanassov, K.; Szmidt, E.; Kacprzyk, J.; Vassilev, P. On intuitionistic fuzzy pairs of $n$-th type. Issues Intuit. Fuzzy Sets Gen. Nets 2017, 13, 136-142.

10. Atanassov, K.T.; Vassilev, P. On the Intuitionistic Fuzzy Sets of $n$-th Type. In Advances in Data Analysis with Computational Intelligence Methods; Studies in Computational Intelligence; Gawęda, A., Kacprzyk, J., Rutkowski, L., Yen, G., Eds.; Springer: Cham, Switzerland, 2018; Volume 738, pp. 265-274.

11. Parvathi, R.; Vassilev, P.; Atanassov, K. A note on the bijective correspondence between intuitionistic fuzzy sets and intuitionistic fuzzy sets of $p$-th type. In New Developments in Fuzzy Sets, Intuitionistic Fuzzy Sets, Generalized Nets and Related Topics; SRI PAS IBS PAN: Warsaw, Poland, 2012; Volume 1, pp. 143-147.

12. Vassilev, P.; Parvathi, R.; Atanassov, K. Note on intuitionistic fuzzy sets of $p$-th type. Issues Intuit. Fuzzy Sets Gen. Nets 2008, 6, 43-50.

13. Atanassov, K. Temporal Intuitionistic Fuzzy Sets; Comptes Rendus de l'Academie bulgare des Sciences; Bulgarian Academy of Sciences: Sofia, Bulgaria, 1991; pp. 5-7.

14. Atanassov, K.; Szmidt, E.; Kacprzyk, J. On intuitionistic fuzzy multi-dimensional sets. Issues Intuit. Fuzzy Sets Gen. Nets 2008, 7, 1-6.

15. Gorzalczany, M. Interval-valued fuzzy fuzzy inference method-Some basic properties. Fuzzy Sets Syst. 1989, 31, 243-251. [CrossRef]

16. Atanassov, K. Review and New Results on Intuitionistic Fuzzy Sets. 1988, IM-MFAIS-1-88, Mathematical Foundations of Artificial Intelligence Seminar. Available online: http:/ /www.biomed.bas.bg/ bioautomation/2016/vol_20.s1/files/20.s1_03.pdf (accessed on 13 July 2018 )

17. Atanassov, K. Review and new results on intuitionistic fuzzy sets. Int. J. Bioautom. 2016, 20, S7-S16. 
18. Atanassov, K.; Gargov, G. Interval valued intuitionistic fuzzy sets. Fuzzy Sets Syst. 1989, 31, $343-349$. [CrossRef]

19. Atanassov, K. Geometrical Interpretation of the Elements of the Intuitionistic Fuzzy Objects. 1989, IM-MFAIS-1-89, Mathematical Foundations of Artificial Intelligence Seminar. Available online: http: / /biomed.bas.bg/bioautomation/2016/vol_20.s1/files/20.s1_05.pdf (accessed on 13 July 2018)

20. Atanassov, K. Geometrical interpretation of the elements of the intuitionistic fuzzy objects. Int. J. Bioautom. 2016, 20, S27-S42.

21. Atanassov, K. Intuitionistic fuzzy logics as tools for evaluation of Data Mining processes. Knowl.-Based Syst. 2015, 80, 122-130. [CrossRef]

(C) 2018 by the author. Licensee MDPI, Basel, Switzerland. This article is an open access article distributed under the terms and conditions of the Creative Commons Attribution (CC BY) license (http:/ / creativecommons.org/licenses/by/4.0/). 